\title{
Physical and Numerical Analysis of Bioclimatic Strategies Implemented on Social Housing
}

Cesar Augusto Real-Ramirez ${ }^{1}$, Jose Maria Velazquez-Soto ${ }^{2}$, Rosalba Orduña-Martinez ${ }^{1}$, Jesus Isidro Gonzalez-Trejo ${ }^{1}$

${ }^{1}$ Systems Department, Universidad Autonoma Metropolitana Azcapotzalco, Distrito Federal, Mexico.

${ }^{2}$ Natural Environment Department, Universidad Autonoma Metropolitana Azcapotzalco, Distrito Federal, Mexico.

Corresponding author: Cesar Augusto Real Ramirez, e-mail: carr@azc.uam.mx.

\begin{abstract}
This paper presents the results of aerodynamical performance research focused on maintaining the thermal comfort and increasing the energy efficiency of a typical social housing unit located in a high-density urban area. Bioclimatic design strategies are used to develop a sustainable and economic technology in existing housing clusters in Mexico City. A full-scale prototype, built on campus facilities, was used to study the flow conditions around the design. All scaled prototypes implement similar criterion. Furthermore, a scaled prototype is evaluated within a low speed wind tunnel installation. Additionally, numerical simulations were performed at transient state based on previous physical measurements and historical local climatic conditions to determine preferable modifications.
\end{abstract}

\section{Keywords}

Bioclimatic design; climatic conditions; wind tunnel; numerical methods; social housing; energy efficiency

\section{Introduction}

Bioclimatic design focuses on natural ventilation and climate control systems with low energy consumption, where the external geometry of the architectural space provides the flow and temperature conditions in which thermal comfort remains constant even if the weather conditions change. Local prevailing winds can be used to construct the architectural spaces in a way that promotes the movement of air mass and minimizes stagnant air volumes inside the housing units based on the interaction of the air with the floor and the facades of the housing. 
Traditionally, architecture has focused on comfort; however, it is necessary to introduce new strategies to generate ideas that lead to design transformation and innovative construction projects. Mexico City is one of the largest metropolitan areas in the world and has a large number of people on the periphery of the urban environment [1].

Historically, human settlements developed with no concept of sustainability. The result is that the total number of hours of transportation and utility costs increased. Providing infrastructure to these high-density populated areas is difficult [2].

In recent decades, politics propitiated urbanization with almost no land cost. The result is a large amount of housing, approximately 3000 units in each cluster without connectivity, which is significantly less sustainable. Mexico County borders the metropolitan area and has a different climate because the mountain system there has many altitudes, as shown in Figure 1.

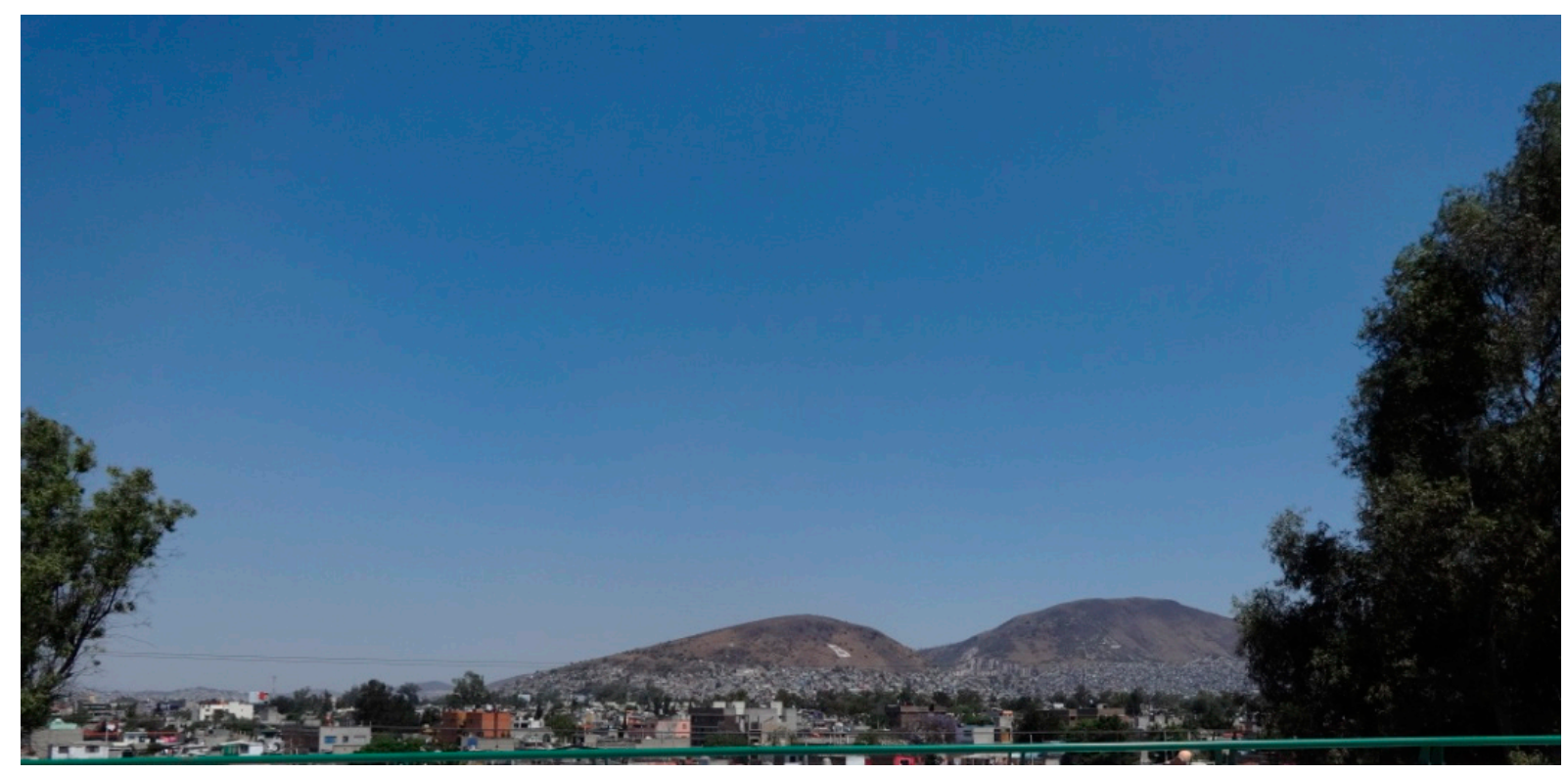

Figure 1. Flat housing in the Mexico City metropolitan area

The height of the buildings is predominantly uniform. Most of the buildings have a least three floors, and they can be described as a "flat city." However, zones exist in which the economic development is proportional to the height of the buildings. This situation is not unique to one city, and in Mexico City, it occurs downtown, in an area known as the country's financial district [3]. 
The flow conditions have no significant differences because of the large density of buildings built in the valley. According to the Systems and Geography National Institute (INEGI), Mexico City, a megalopolis is located in Mexico's Valley with an altitude of $2240 \mathrm{~m}$ above sea level. This valley is in a volcanic belt on the high plateaus of south-central Mexico [4].

Because of the high-density of people in the valley, the heat release mechanism is intensified, as human activities and additional greenhouse gases being emitted to the atmosphere. In the case of large cities, urbanization creates a microclimate. In the case of Mexico City, the air temperature is approximately 5 degrees above the local temperature.

The first part of the study is based on physical and numerical simulation of the flow conditions around an approved prototype housing unit based on the federal government standard construction requirements within the Valley of Mexico [5]. Physical simulations were performed using a fully scaled prototype constructed within the Universidad Autonoma Metropolitana (UAM) campus facilities in an urban environment.

The inlet flow conditions toward the housing unit are important because they determine whether the environment is urban or semi-urban. The topography around the housing, solar trajectories, rainfall, vegetation, magnitude and trajectory of the wind all contribute to user comfort [6].

The climatic area of the housing is very important. Cool climates need to have solar gain and be dry, warm climates where solar radiation needs to be minimized. Mexico City has a subtropical highland climate based on the Köppen climate classification [7]. The average annual temperature of the metropolitan area varies from $12^{\circ}$ to $16^{\circ} \mathrm{C}$. Specific months and hours of the day can provide direct thermal comfort within the housing unit without air conditioning [8-10].

A local climatic station was installed at the top of one of the buildings around the housing. This allowed us to obtain the predominant flow conditions for the prototype that were then used as inlet conditions within the wind tunnel and in numerical simulations. It was necessary to develop a study where wind speed and wind direction were controlled conditions for aerodynamic tests [11].

According to local measured climatic conditions, the range of wind speed varied between 0 and $5 \mathrm{~m} \cdot \mathrm{s}^{-1}$. A scaled prototype constructed in translucent acrylic was used in a low-speed wind tunnel to reproduce different flow patterns. Urban, semiurban and uniform flow patterns can be used for different purposes [10, 12, 13]. 


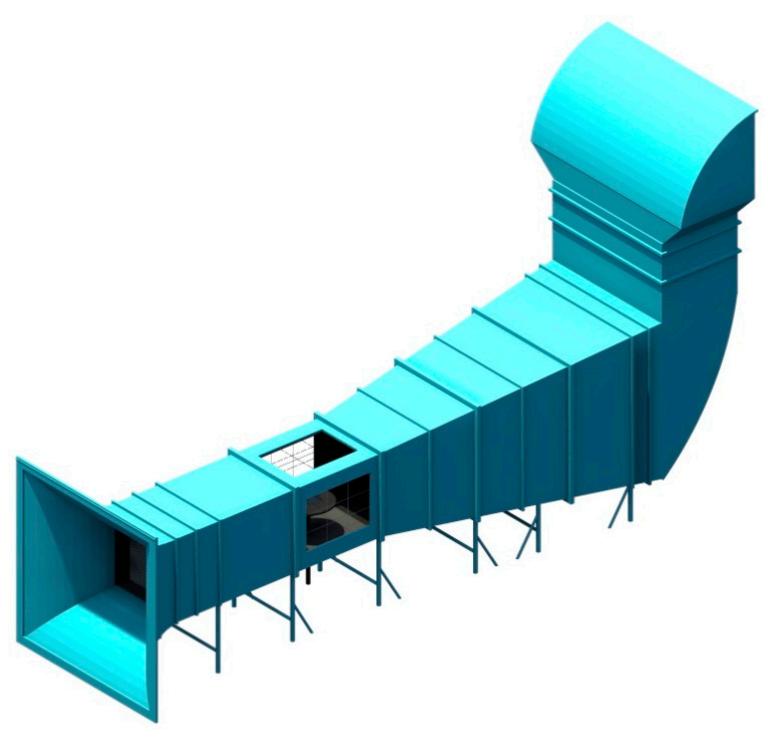

Figure 2. Low speed wind tunnel schematic diagram located at Universidad Autonoma Metropolitana Azcapotzalco

Figure 2 shows a schematic diagram of an open circuit, low speed wind tunnel located at the Universidad Autonoma Metropolitana Azcapotzalco. The wind tunnel is fully characterized and has automated acquisition instruments to register the thermodynamic variables needed for the determination of the velocity profile within the wind tunnel test zone [14].

Local measurements included several thermodynamic variables, for example, air humidity, air velocity magnitude, air direction, outdoor temperature, indoor temperature and atmospheric pressure. They were measured using a climatologic station located on a building close to the fully scaled prototype. 


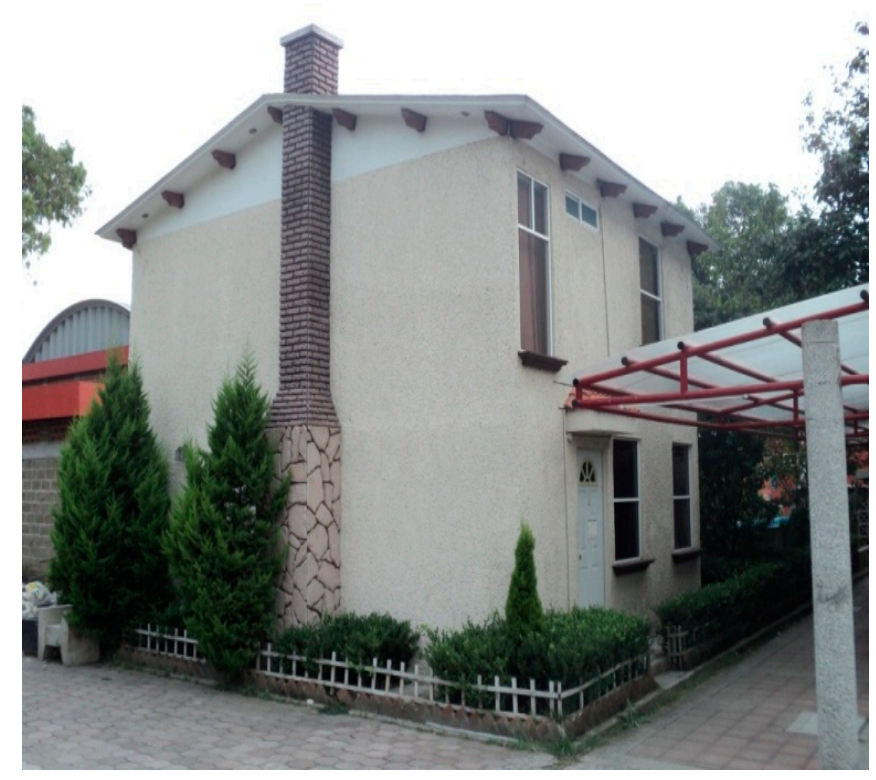

Figure 3. Physical model of the housing unit in compliance with federal government standards

Figure 3 shows the fully scaled prototype that was built in compliance with the Mexican federal government standards at the University campus facilities to simulate different climatic conditions. The housing is oriented to the south, and the figure shows the rear facade of the dwelling. A chimney, bushes and a fence, which are shown, are not considered in the aerodynamic study. Around the housing unit, there are electrical facilities and a casting laboratory with a covered corridor between them. The flow conditions are similar to those we might find in an area with a high-density population. Approximately $150 \mathrm{~m}$ to the north is the main road and a housing area with many six-floor buildings.

Translucent acrylic was used within the wind tunnel to evaluate the aerodynamic performance, quantify the benefit of an integrated proposal and estimate the energy savings because of the benefit in kinetic energy of the air flow.

\section{Physical and numerical model}

The construction of a scaled prototype of social housing must guarantee that the qualitative behavior of the air at different climatic conditions can be repeated. To obtain this similarity, the design must have the same geometry. Despite using a scaled prototype, the ratio between physical dimensions was preserved, and different geometries around the housing, such as windows and doors, were scaled. 
Achieving a full geometric similarity is not practical because some of the design features that should have different parts or accessories have to be analyzed individually to obtain a scaled prototype within the wind tunnel. This design is unique to certain speed regimes. In a subsequent analysis, we will present the contribution of the most important interior housing factors affecting the aerodynamic performance of the dwelling.

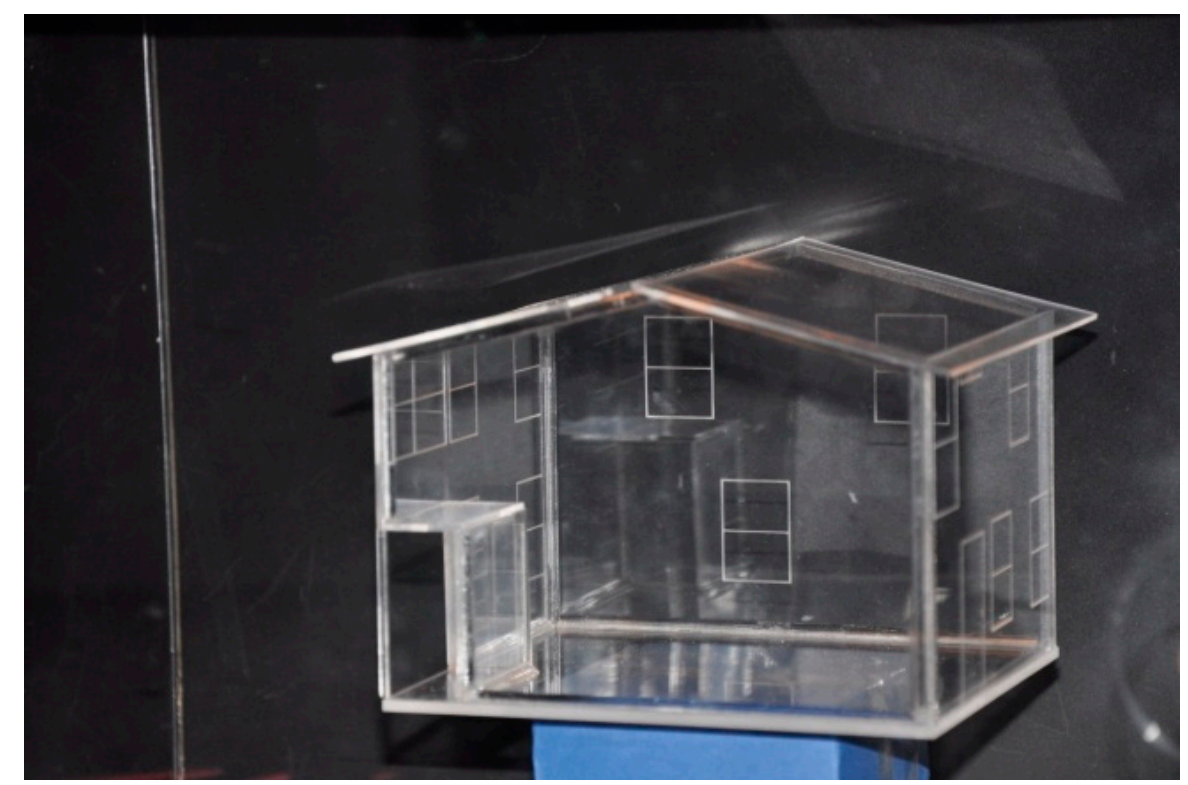

Figure 4. Physical representation of a typical social housing unit in Mexico within the wind tunnel

According to the National Housing Institute in Mexico (INFONAVIT), the average field in social housing is $30 \mathrm{~m}^{2}$. The full-scaled prototype has $80 \mathrm{~m}^{2}$ distributed over two floors, 12 windows and two exterior doors. The restroom and bedrooms are located at the top floor and the dining room, living room and kitchen are in the basement.

Translucent acrylic, $3 \cdot 10^{-2} \mathrm{~m}$ thick, was used in the physical scaled design. No parts were attached, and no unique base was used to change the boundary layers and the air flow toward the scaled model. In Figure 4, the geometric casing of the dwelling is presented. The significant external elements used to achieve geometric similarity are outlined in the model. The fireplace was removed because in the full-scaled prototype, it improved aesthetics but is not considered a constitutive in the final proposal, as shown in Figure 5. The numerical model used the geometric similarity criteria and solved the Navier-Stokes equations for a turbulence model. 


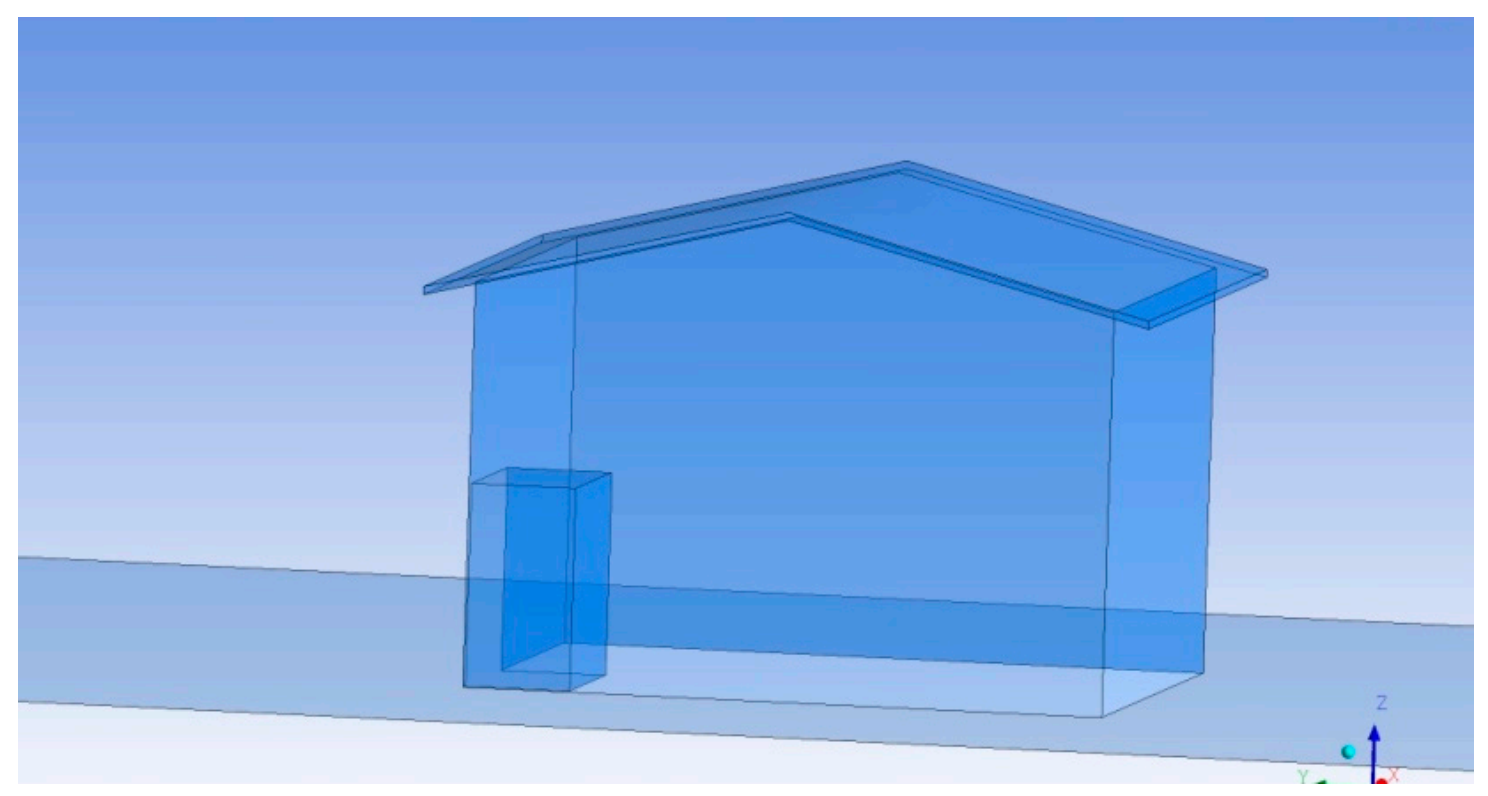

Figure 5. Numerical model of a typical social housing unit in Mexico

This study presents only the fluid flow around the dwelling and does not analyze the interior fluid flow, which is going to be presented in future work. One of the main design criteria in the development process of the proposals was the compliance federal government construction standards [5]. The study also considers cost and ease of installation.

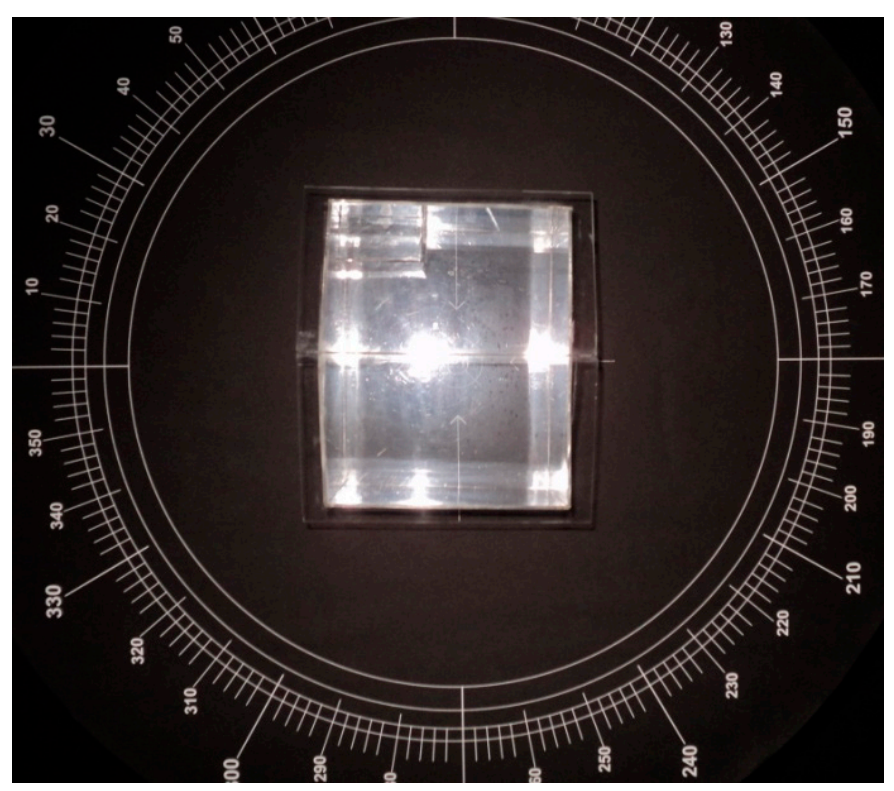

Figure 6. Aerial view of the scaled prototype mounted on a rotating platform within the wind tunnel 
Figure 6 shows the translucent acrylic prototype mounted on a graduated rotating base. This base allows us to know the different orientations in which the wind can reach the exterior walls of the housing. The experimental facility allows us to precisely know the wind direction and visualize it in different planes, such as vertically and horizontally oriented in accordance with the light source used. The diameter of the base contributes to the flow conditions of the physically scaled design because the development of the flow on the surface will be related to the distance between the edge of the base and the housing.

The operation conditions in the laboratory were taken during the test with a meteorological station located on the roof of the installation, which reported a relative humidity of 56\%, the atmospheric pressure of $78000 \mathrm{~Pa}$ and $294.15 \mathrm{~K}$ of local temperature.

Once the flow condition is uniform, a minimum of $15 \mathrm{~min}$ of operation at the same frequency, the model is moved to the predefined area within the wind tunnel known as the test zone to allow a fully developed flow. In previous studies, an urban flow profile was reached with basic geometric elements that modified the flow. Future work will present the characterization of different flow conditions to describe urban and semi-urban patterns within the low-speed wind tunnel.

A future research project will focus on developing models where thermal studies are reported using thermal and optical equipment under different local atmospheric conditions within the low-speed wind tunnel. The goal of this study will be to reproduce the flow profile conditions in the principal regions of the country and a future publication will present the progress of this research.

\section{Methodology}

The constitutive equations of the flow surrounding the dwellings are the Navier-Stokes and continuity equations. Both are presented in vector form.

$$
\begin{gathered}
\rho \frac{D \vec{v}}{D t}=-\nabla p+\mu \nabla^{2} \vec{v}+\rho \vec{g} \\
\frac{\partial \rho}{\partial t}+(\nabla \bullet \rho \vec{v})=0
\end{gathered}
$$

The turbulence model used in this study is the Large Eddy Simulation (LES) model, which is solved numerically using commercial software named Fluent [15]. In an LES turbulence model, the Navier-Stokes equations are filtered, and the 
numerical model calculates two conditions, which are the vortex's influence due to mesh size and the vortexes smaller than the mesh size.

The filtered variable is defined by the following equation:

$$
\bar{\varphi}(x)=\int_{D} \varphi\left(x^{\prime}\right) G\left(x, x^{\prime}\right) d x^{\prime}
$$

where $\bar{\phi}(x)$ is the stresses deformation, D is the flow domain, and $\mathrm{G}$ is the filtered function that determines the scale of the resolved vortexes. If we discretize equation (3) over a finite volume, we obtain the following equation:

$$
\bar{\varphi}(x)=\frac{1}{V} \int_{V} \varphi\left(x^{\prime}\right) d x^{\prime}, x^{\prime} \in V
$$

where $\mathrm{V}$ is the volume of the computational cell. The filtered function $G\left(x, x^{\prime}\right)$ implies two conditions, $\frac{1}{V}, x^{\prime} \in v$ and $0, x^{\prime}$ for everything else. The flow conditions in this study require that the air is considered incompressible resulting in the LES turbulence model equations described below:

$$
\begin{gathered}
\frac{\partial \rho}{\partial t}+\frac{\partial}{\partial x_{i}}\left(\rho \bar{u}_{i}\right)=0 \\
\frac{\partial}{\partial t}\left(\rho \bar{u}_{i}\right)+\frac{\partial}{\partial t}\left(\rho \bar{u}_{i} \bar{u}_{j}\right)=\frac{\partial}{\partial x_{j}}\left(\mu \frac{\partial \bar{u}_{i}}{\partial x_{j}}\right)-\frac{\partial \bar{p}}{\partial x_{i}}-\frac{\partial \tau_{i j}}{\partial x_{j}}
\end{gathered}
$$

The tensor data calculated in the sub-grid area is developed from the Smagorinsky-Lilly model and the discretized equation is as follows:

$$
\tau_{i j} \equiv \rho \overline{u_{i} u_{j}}-\rho \overline{u_{i} u_{j}}
$$

The main advantages of the LES turbulence model are as follows. (i) It is possible to reproduce the transient flow pattern around the housing that is needed to study the proposal. (ii) It considers an approximation of the larger scale vortexes that are calculated in the numerical simulation and use a set of filtered equations. The turbulence is filtered and allows for solution of a greater number of unknowns and reduces numerical error. (iii) The accuracy of the results needs to be compared with physical experiments that cannot be solved using other turbulence models. 
The computing resources used in LES are higher than DES, RSM, k-e and laminar models. In some cases, the steady state solution is not possible because of the boundary conditions, nature of phenomenon, convergence time and available computational. The most significant disadvantages of using an LES turbulence model are as follows. (i) The accuracy on the results of fluid variables could not be validated. (ii) The application of LES is valid only for three-dimensional cases and in nonsteady state. (iv) It is not possible to use multiphase flows.

\section{Results}

To obtain reliable results of physical experiments, we considered that the orientation of the housing, layout of the surrounding buildings, wind direction and the main road $150 \mathrm{~m}$ away from the facility could locally modify the direction, speed and magnitude of the wind to east-west and from 0 to $5 \mathrm{~m} \cdot \mathrm{s}^{-1}$.

In physical experiments within the wind tunnel, it was possible to obtain repeatability at different operational conditions. In accordance with historical information about climatic conditions, the velocity magnitude was set at $4 m \cdot s^{-1}$ in the northeast direction. The information obtained with the previously mentioned local climatic station was processed and used as an inlet condition for numerical simulations.

Flow visualization was performed using continuously produced white smoke, which was injected at the entrance of the open circuit wind tunnel to reproduce flow features for the scaled model. A class IIla, $650 \mathrm{~nm}, 2.5 \mathrm{~mW}$, red diode laser was used to illuminate a vertical plane to highlight the three-dimensional features that develop around the design, as shown in Figure 7. 


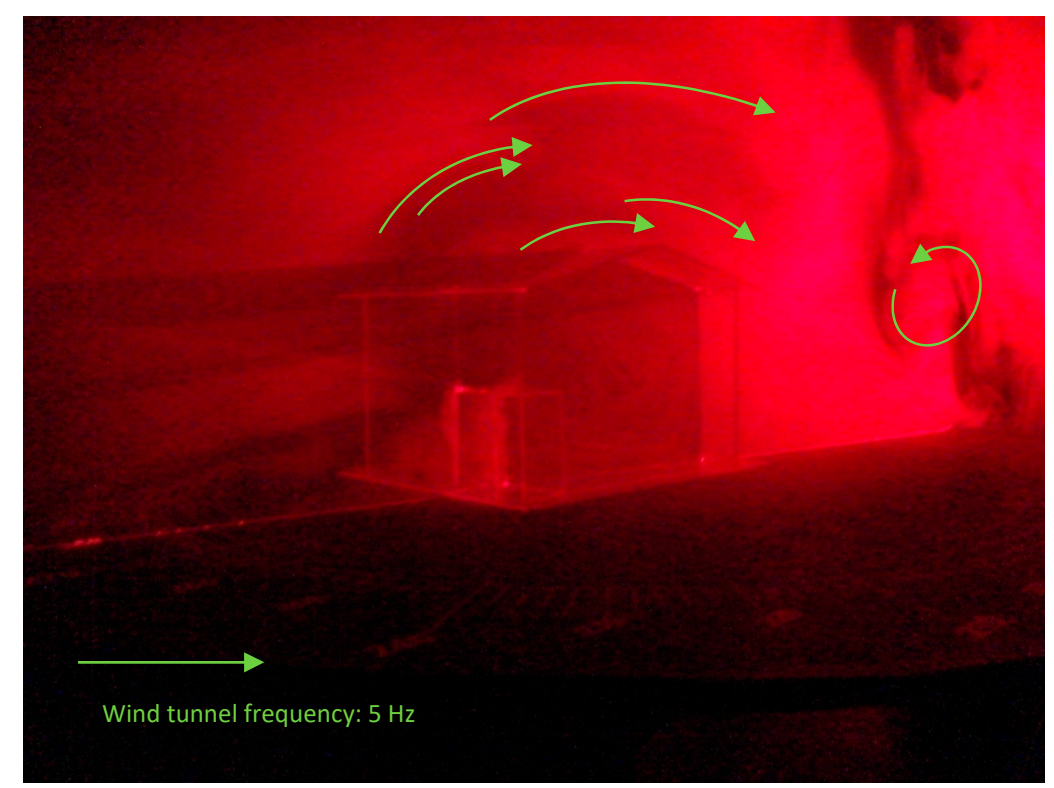

Figure 7. Isometric view and laser illuminated plane of the case study

According to Meza, a $5 \mathrm{~Hz}$ change in the frequency control is equivalent to $0.5 \mathrm{~m} \cdot \mathrm{s}^{-1}$ in the wind speed magnitude within the test zone of the tunnel. In this study, we determined the test zone and ensured that the velocity profile is fully developed and repeatable [14]. To satisfy the dynamic similarity, we scaled the velocity magnitude of the working fluid based on $5 \mathrm{~Hz}$ changes in the wind tunnel frequency control. Once in the wind tunnel, the air enters a region with a flow straightening honeycomb geometry, which ensures that the flow in the test area is uniform and laminar.

A mixture of water-based lubricant was heated to generate white smoke that has a similar density to air and did not modify the inlet flow upon injection. Laser based lighting allowed us to study the flow behavior using different vertical planes, and subsequently to interpret the three-dimensional behavior of the flow. As shown in Figure 7, the air impacts the rear wall of the prototype and divides the flow, with resulting upward and downward flow directions.

Downward flow swirls with the walls of the housing and drives the surrounding upward flow. The upward flow collides with the top cover of the housing and is mainly diverted into two directions, left and right. Figure 7 illustrates the phenomenon with green arrows.

Once the flow passes through the housing, it interacts with the front wall of the prototype and the floor and the air becomes turbulent. This action develops at a distance of at least five times the length of the housing and is relative to wind speed. This 
flow condition repeats continuously and is known as an urban environment, which may be affected by different housing designs.

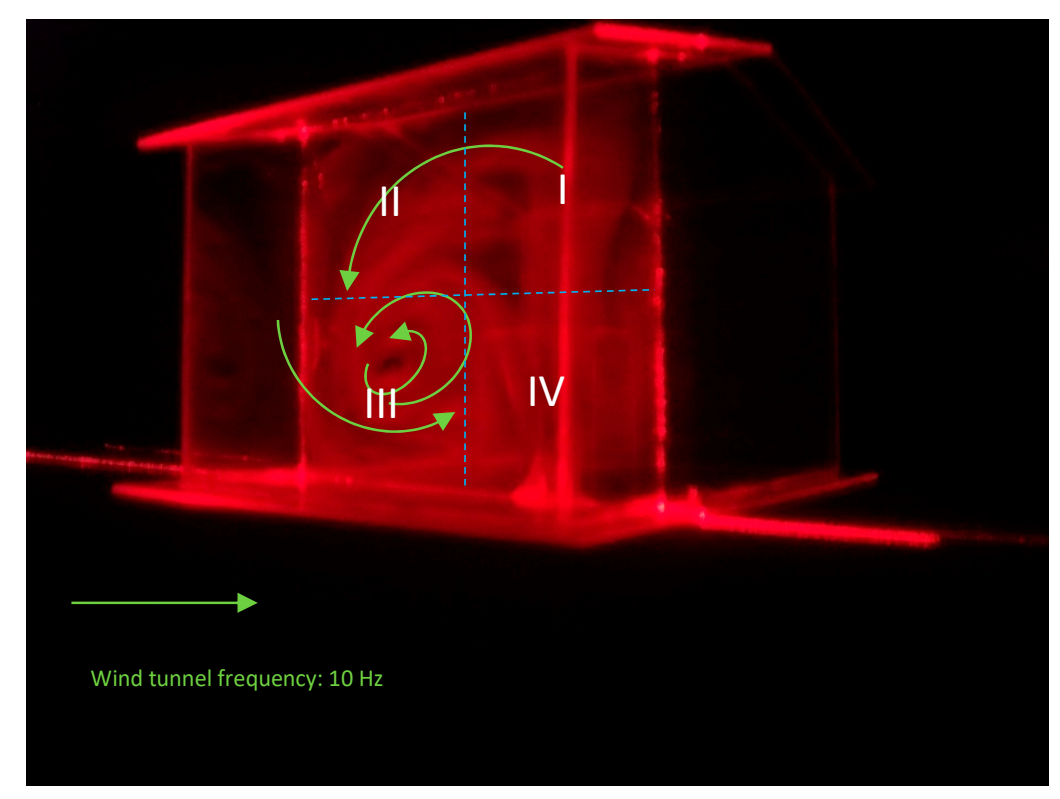

Figure 8. Laser illuminated and quadrant delimited plane for internal volume of the study case

The laser illumination allowed us to study "virtual" planes that helped to interpret the outside and inside flow around an architectural design within a wind tunnel. As shown in Figure 8, a pulse of smoke entered the design without interior architectural elements to observe the possible interaction of interior and exterior flow. The kinetic energy of the vortexes, generated from the interaction with the walls of the house, distributed around the internal volume and generated streams, but there was a preponderant direction in swirl. Quadrants were used to divide the plane, which allowed us to determine two different vortexes. Both had the same rotation; however, in quadrant three, there was a smaller and more intense vortex. The second vortex, located in the first quadrant, covered nearly the entire area of the plane, but it did not have enough energy to promote thermal comfort because of the low speed magnitude of the air. 


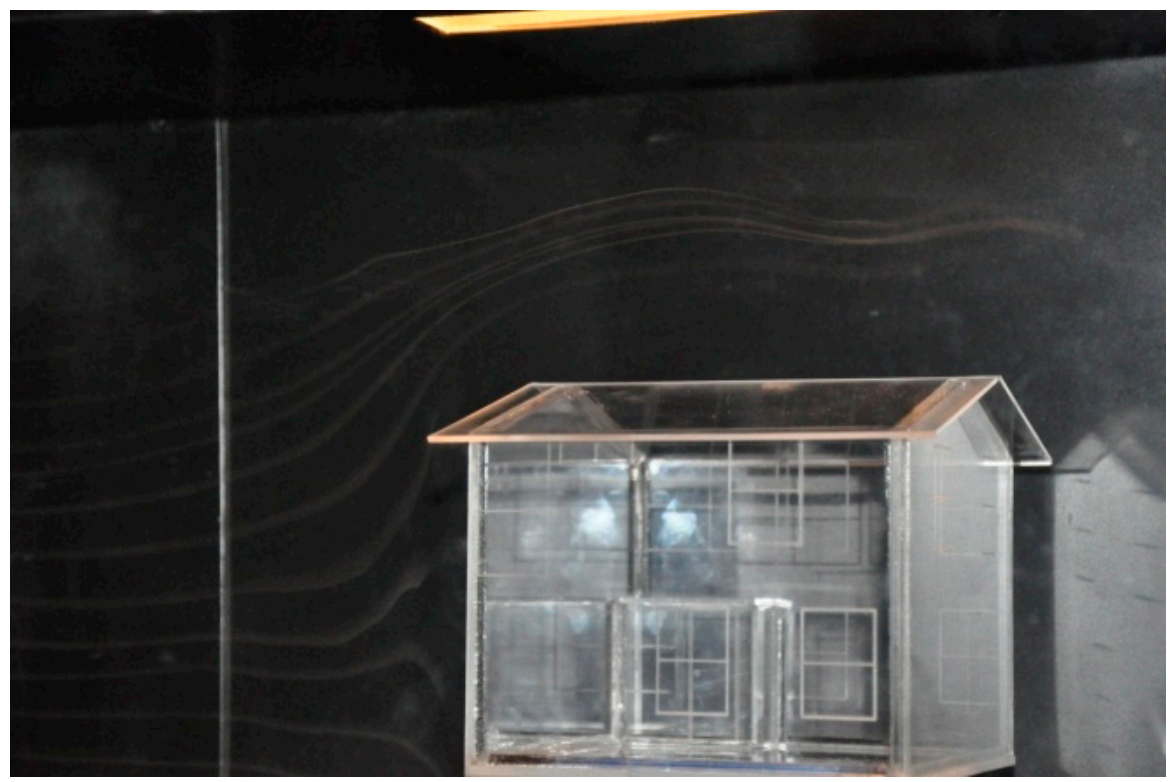

Figure 9. Aerodynamic performance of the scaled model within the wind tunnel

Figure 9 shows eleven stream traces generated through a smoke comb machine. A metal filament burned liquid oil at different equidistant heights of the tube and produced a continuous line that moved because of the air inside the wind tunnel.

Figure 8 and Figure 9 have differences. The white streamlines describe a zone above the housing, which is primarily because of the interaction of the flow with the roof. This phenomenon affects the heat transfer to the outside because it generates a natural thermal insulator that prevents natural convection because of the prevailing winds.

Physical simulations validated the numerical simulations. As shown in Figure 10, air behavior is reproduced qualitatively and lead to an interpretation of the recirculation zone previously mentioned. When the flow split, above the upper cover of the housing, the recirculation zone was equal length to the housing. Both visualization techniques and numerical simulations indicate that turbulent flow is obtained because of the interaction with the walls, the boundary layer than generate between the upper cover and the flow and is enhanced because of the upper flow. 


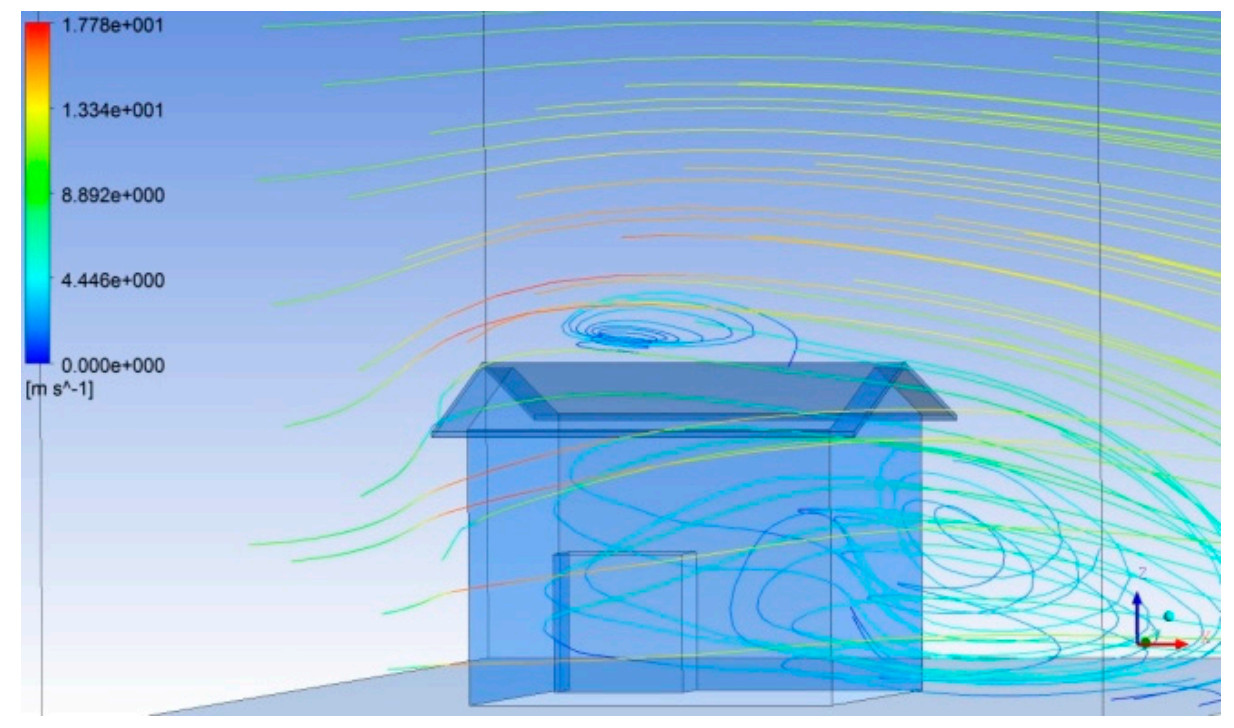

Figure 10. Numerical simulation results of aerodynamic performance of social housing

The modification of the surface roughness in the upper cover can reduce the development of the boundary layer. This study proposes the use of "steps" to affect the growth of the layer and increase the convection heat transfer. In the past, this concept was successfully implemented. Over the last century, Central America housing used "pavers" in upper covers to maintain interior comfort in regions close to Ecuador. Several authors have studied this technology [16, 17]; however, our proposal suggests the installation of solar cells inside the bricks, from which energy reserves can be measured [18-20].

The numerical model reproduced the flow pattern that developed in the wind tunnel experiment. The pattern consisted of three main streams of convergence: the downward flow of the top cover and the two side streams, left and right paths. The recirculation zone begins from the side walls of the housing. Another observation is that the minimum speed of the stream can be close to zero. Figure 10 indicates that the kinetic energy of the air is not affected by the height of the house and is principally in the stream above the housing.

The three-dimensional flow pattern is shown in Figure 11 and 12. The experimental method, which used the smoke machine, preferentially described laminar flow regions. In the physical experiment, the turbulent flow pattern quickly dissipates particles from the oil combustion. The visualization allowed us to identify regions of turbulent flow around the design and show that the mixing process occurs more intensively. 


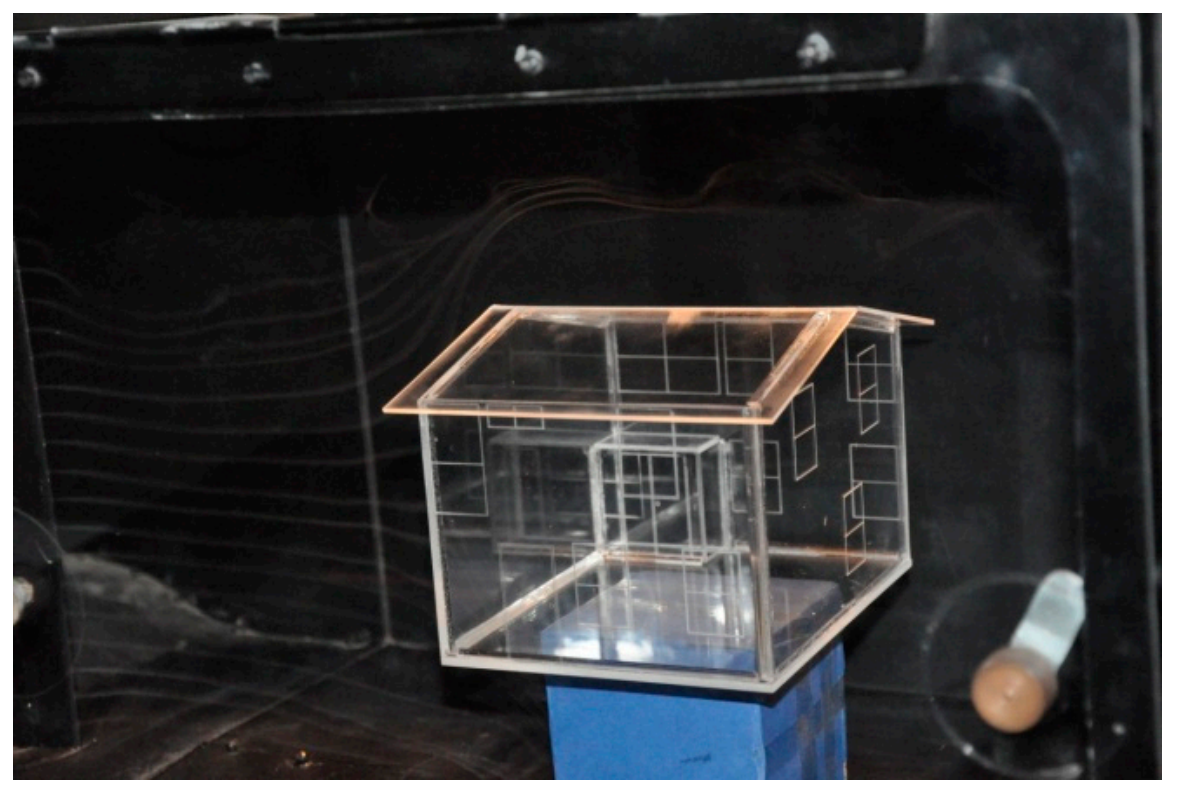

Figure 11. Inlet flow profile for the scaled physical model

Therefore, we suggest that the laminar flow pattern occurs under controlled conditions within the wind tunnel and can be considered an ideal profile, as shown in Figure 11. In this study, the mixing process occurs between four main streams that form around the model. This process affects the convection and the thermal comfort inside the building.

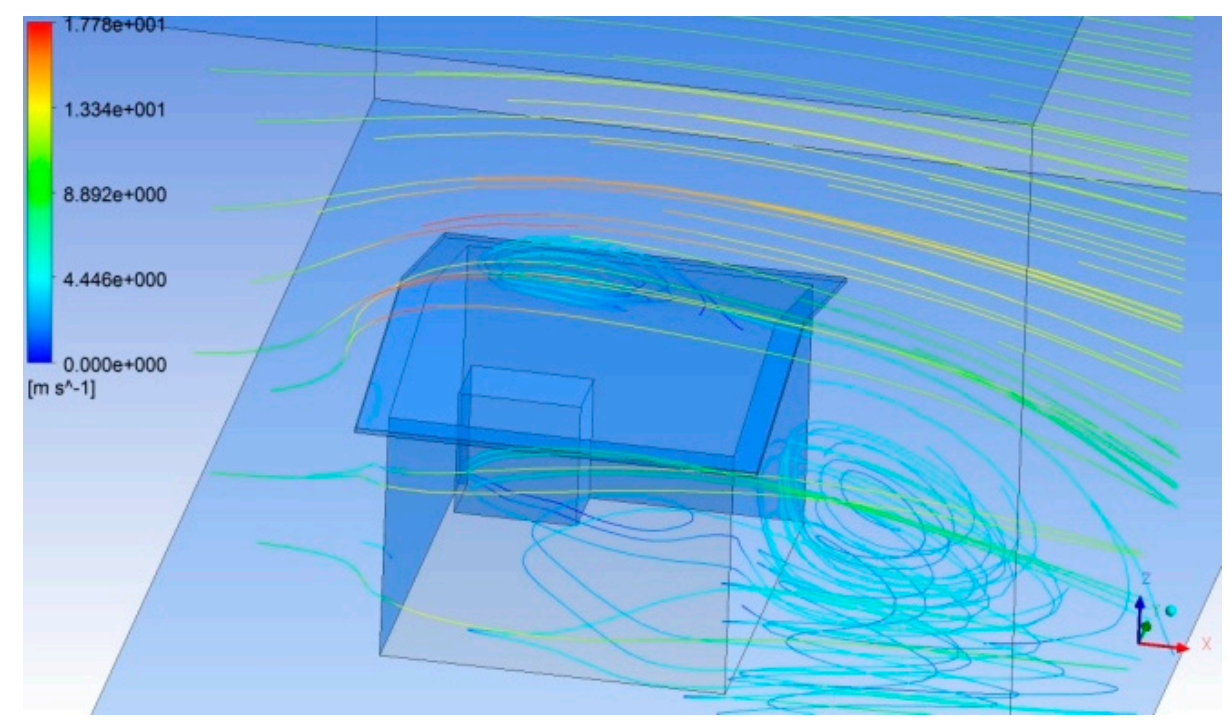

Figure 12. Numerical simulation results for the inlet flow profile 
Once validated, the numerical simulations can be useful tools that allow one to know and interpret the three-dimensional nature of the phenomenon and its complexity. Numerical simulations passed through a refinement mesh process and a convergence analysis was performed. The results show that there is no symmetry in the flow pattern. This phenomenon occurs in the upper cover and in the turbulence zone at the front of the housing. As shown in Figure 12, recirculation zones can be clearly identified because the air speed is closed to zero, forming a volume with the same direction of swirl. The flow condition on the surfaces is close to zero because of the boundary condition and has no surrounded swirl.

\section{Conclusions}

The external geometry of a housing unit determines the characteristics of the flow pattern, and its surroundings on a large scale can change the surrounding environment. The bioclimatic design presents strategies with passive design elements to reduce energy consumption and to maintain a constant temperature. The use air's kinetic energy can decrease the total energy consumption in social housing. The most conservative value mentioned is five percent. This amount may seem negligible, but in social interest housing sectors, it represents meaningful savings. The proposed modifications can be made with inexpensive technology.

\section{Acknowledgments}

The work described in this paper was supported by research grants from Consejo Nacional de Ciencia y Tecnologia (CONACYT) and the Research Grants of the Universidad Autonoma Metropolitana. Numerical and physical simulations were performed in the Scientific Computing and Visualization Laboratory in the Systems Department of Universidad Autonoma Metropolitana Azcapotzalco. Statistical data were obtained from the National Geography and Statistical Institute of Mexico (INEGI).

\section{References}

[1] INEGI, Síntesis metodológica y conceptual del Censo de Población y Vivienda 2010, in: I.N.d.E.y. Geografía (Ed.) Diseño de la muestra censal 2010, Instituto Nacional de Estadística y Geografía, Mexico, 2010, pp. 81. 
[2] INEGI, Electricidad, agua y suministro de gas por ductos al consumidor final. Censos Económicos 2004, in: INEGI (Ed.), INEGI, Mexico, 2006, pp. 93.

[3] D.E. Davis, Zero-Tolerance Policing, Stealth Real Estate Development, and the Transformation of Public Space Evidence from Mexico City, Lat Am Perspect, 40 (2) (2013) 53-76.

[4] F. Maza, Diccionario Porrua de Historia, Biografia y Geografia de Mexico, 6th ed., Porrua, Mexico, 1995.

[5] CONAVI, Código de Edificación de Vivienda, in: G. Federal (Ed.), CONAVI, Mexico, 2010.

[6] W.B. Fitzgerald, M. Fahmy, I.J. Smith, M.A. Carruthers, B.R. Carson, Z. Sun, M.R. Bassett, An assessment of roof space solar gains in a temperate maritime climate, Energ Buildings, 43 (7) (2011) 1580-1588.

[7] P. Connolly, Observing the evolution of irregular settlements Mexico City's colonias populares, 1990 to 2005, Int Dev Plann Rev, 31 (1) (2009) 1-35.

[8] A. Chel, G.N. Tiwari, Stand-alone photovoltaic (PV) integrated with earth to air heat exchanger (EAHE) for space heating/cooling of adobe house in New Delhi (India), Energ Convers Manage, 51 (3) (2010) 393-409.

[9] M.A. Tahat, H. Al-Hinai, S.D. Probert, Performance of a low-energy-consumption house experiencing a Mediterranean climate, Appl Energ, 71 (1) (2002) 1-13.

[10] K. Richards, T.R. Oke, Validation and results of a scale model of dew deposition in urban environments, Int J Climatol, 22 (15) (2002) 1915-1933.

[11] J.G. Tsutsumi, Effects of the wind on thermal sensation in a house with natural ventilation, Wind Engineering into the 21st Century, Vols 1-3, (1999) 1959-1964.

[12] A.H. Rosenfeld, H. Akbari, S. Bretz, B.L. Fishman, D.M. Kurn, D. Sailor, H. Taha, Mitigation of Urban Heat Islands - Materials, Utility Programs, Updates, Energ Buildings, 22 (3) (1995) 255-265.

[13] M. Dewsbury, T. Chandler, Massive timber as effective thermal mass in Australian contemporary housing, Living and Learning: Research for a Better Built Environment, (2015) 382-392. 
[14] A.E. Fernandez-Meza, Caracterizacion del túnel aerodinámico del laboratorio de bioclimática de la Universidad Autónoma Metropolitana: como herramienta para estudios de modelos arquitectónicos, Medio Ambiente, Universidad Autonoma Metropolitana, 2008.

[15] I. Fluent, Fluent 6.3 Theory Guide, in, Ansys Inc, 2006.

[16] Y. Wang, E.S. Long, S.M. Deng, Applying passive cooling measures to a temporary disaster-relief prefabricated house to improve its indoor thermal environment in summer in the subtropics, Energ Buildings, 139 (2017) 456464.

[17] Y. Wang, L. Wang, E.S. Long, S.M. Deng, An experimental study on the indoor thermal environment in prefabricated houses in the subtropics, Energ Buildings, 127 (2016) 529-539.

[18] V. Kapsalis, D. Karamanis, On the effect of roof added photovoltaics on building's energy demand, Energ Buildings, 108 (2015) 195-204.

[19] M.H. Chung, J.C. Park, M.J. Ko, Effect of the solar radiative properties of existing building roof materials on the energy use in humid continental climates, Energ Buildings, 102 (2015) 172-180.

[20] P.J. Rosado, D. Faulkner, D.P. Sullivan, R. Levinson, Measured temperature reductions and energy savings from a cool tile roof on a central California home, Energ Buildings, 80 (2014) 57-71. 